\title{
Properties of the Discrete Hilbert Transform
}

\author{
Rashid A. Aliev ${ }^{1,2}$. Aynur F. Amrahova1,3 \\ Received: 11 December 2017 / Accepted: 10 June 2019 \\ ๑) Springer Nature Switzerland AG 2019
}

\section{Abstract}

The asymptotic behavior of the distribution function of the Hilbert transform of sequences from the class $l_{1}$ is studied. The concept of $Q$-summability of series is introduced; using this notion, it is shown that the Hilbert transform of a sequence from the class $l_{1}$ is $Q$-summable and is $Q$-sum is zero.

Keywords Discrete Hilbert transform - Asymptotic behavior of the distribution function · Q-integral · Q-summability

Mathematics Subject Classification 44A15 · 26A39 • 40G99

\section{Introduction}

Let $\left\{b_{n}\right\}_{n \in Z} \in l_{1}$. The sequence

$$
\tilde{b}_{n}=\sum_{m \neq n} \frac{b_{m}}{n-m}, \quad n \in Z,
$$

is called the Hilbert transform of the sequence $\left\{b_{n}\right\}_{n \in Z}$.

M. Riesz (see [18, see also 10,15$]$ ) proved that if $\left\{b_{n}\right\}_{n \in Z} \in l_{p}, p>1$, then $\left\{\tilde{b}_{n}\right\}_{n \in Z} \in l_{p}$ and the inequality

$$
\left\|\tilde{b}_{n}\right\|_{p} \leq C_{p}\left\|b_{n}\right\|_{p}
$$

Communicated by Vladimir Bolotnikov.

$凶 \quad$ Rashid A. Aliev

aliyevrashid@mail.ru

Aynur F. Amrahova

amrahovaaynur@mail.ru

1 Baku State University, 1148 Baku, Azerbaijan

2 Institute of Mathematics and Mechanics, NAS of Azerbaijan, 1141 Baku, Azerbaijan

3 Khazar University, 1096 Baku, Azerbaijan 
holds. Weighted analogues of (1) are investigated in the works [7-9, 13, 14, 16, 17, 21].

If $\left\{b_{n}\right\}_{n \in Z} \in l_{1}$, then the sequence $\left\{\tilde{b}_{n}\right\}_{n \in Z}$ belong to the class $\bigcap_{p>1} l_{p}$, but it does not belong to the class $l_{1}$. In this case, R. Hunt, B. Muckenhoupt and R. Wheeden (see [14]) proved that the distribution function $\tilde{b}(\lambda)=\sum_{\left\{n \in Z:\left|\tilde{b}_{n}\right|>\lambda\right\}} 1$ of the Hilbert transform of the sequence $\left\{b_{n}\right\}_{n \in Z}$ satisfies the condition

$$
\forall \lambda>0:|\tilde{b}(\lambda)| \leq \frac{C_{0}}{\lambda} \sum_{n \in Z}\left|b_{n}\right|,
$$

where $C_{0}$ is an absolute constant. Note that for the sequence $\left\{b_{n}\right\}_{n \in Z} \in l_{1}$ the series $\sum_{n \in Z} \tilde{b}_{n}$ does not converge even in the sense of the principal value, i.e., in the sense

$$
\sum_{n \in Z} \tilde{b}_{n}=\lim _{N \rightarrow \infty} \sum_{|n| \leq N} \tilde{b}_{n} .
$$

In the present paper, we study the asymptotic behavior of the distribution function $\tilde{b}(\lambda)$ of the Hilbert transform of a sequence $\left\{b_{n}\right\}_{n \in Z} \in l_{1}$ as $\lambda \rightarrow 0$ (Theorem 1). We introduce the concept of $Q$-summability of series and, using this notion, prove that the Hilbert transform of a sequence $\left\{b_{n}\right\}_{n \in Z} \in l_{1}$ is $Q$-summable and its $Q$-sum is zero (Theorem 2).

\section{Asymptotic Behavior of the Distribution Function of the Discrete Hilbert Transform}

Theorem 1 Let $\left\{b_{n}\right\}_{n \in Z} \in l_{1}$. Then

$$
\lim _{\lambda \rightarrow 0+} \lambda \cdot \tilde{b}(\lambda)=2\left|\sum_{n \in Z} b_{n}\right|,
$$

where $\tilde{b}(\lambda)=\sum_{\left\{n \in Z:\left|\tilde{b}_{n}\right|>\lambda\right\}} 1$ is the distribution function of the Hilbert transform of $\left\{b_{n}\right\}_{n \in Z}$.

We first prove the auxiliary lemma.

Lemma 1 Let $\left\{b_{n}\right\}_{n \in Z} \in l_{1}$ and $\sum_{n \in Z} b_{n}=0$. Then

$$
\tilde{b}(\lambda)=o\left(\frac{1}{\lambda}\right), \lambda \rightarrow 0+.
$$

Proof of Lemma 1 We first assume that a sequence $\left\{b_{n}\right\}_{n \in Z} \in l_{1}$ is concentrated on some finite interval $[-m, m]$, that is, $b_{n}=0$ for $|n|>m$. For every $|k|<m$ and $|n|>2 m$, we have 


$$
|n-k| \geq|n|-|k|>|n|-m>|n|-\frac{|n|}{2}=\frac{|n|}{2}, \quad\left|n-\frac{1}{2}\right| \geq|n|-\frac{1}{2} \geq \frac{|n|}{2} .
$$

Therefore, in this case, for $|n|>2 m$ from the equality

$$
\begin{aligned}
\tilde{b}_{n} & =\sum_{k \neq n} \frac{b_{k}}{n-k}=\sum_{|k| \leq m} \frac{b_{k}}{n-k}=\sum_{|k| \leq m} \frac{b_{k}}{n-k}-\frac{1}{n-1 / 2} \sum_{k \in Z} b_{k} \\
& =\sum_{|k| \leq m} \frac{b_{k}}{n-k}-\frac{1}{n-1 / 2} \sum_{|k| \leq m} b_{k}=\sum_{|k| \leq m} \frac{k-1 / 2}{(n-k)(n-1 / 2)} b_{k}
\end{aligned}
$$

we obtain

$$
\left|\tilde{b}_{n}\right| \leq \sum_{|k| \leq m} \frac{|k-1 / 2|}{|n-k||n-1 / 2|} b_{k} \leq \frac{4}{n^{2}} \sum_{|k| \leq m}|k-1 / 2|\left|b_{k}\right|
$$

Denote

$$
M_{0}=\sum_{|k| \leq m}|k-1 / 2|\left|b_{k}\right|
$$

Then it follows from (5) that

$$
\begin{aligned}
& \left\{n \in Z:\left|\tilde{b}_{n}\right|>\lambda\right\} \subset\{n \in Z:|n| \leq 2 m\} \cup\left\{n \in Z \backslash[-2 m, 2 m]: \frac{4}{n^{2}} M_{0}>\lambda\right\} \\
& =\{n \in Z:|n| \leq 2 m\} \cup\left\{n \in Z \backslash[-2 m, 2 m]:|n|<2 \sqrt{M_{0} / \lambda}\right\} .
\end{aligned}
$$

Hence we have

$$
\tilde{b}(\lambda)=\sum_{\left\{n \in Z:\left|\tilde{b}_{n}\right|>\lambda\right\}} 1 \leq \sum_{\{n \in Z:|n| \leq 2 m\}} 1+\sum_{\left\{n \in Z \backslash[-2 m, 2 m]:|n|<2 \sqrt{M_{0} / \lambda}\right\}} 1 \leq 4 m+4 \sqrt{M_{0} / \lambda}+2,
$$

whence the asymptotic Eq. (4) follows.

Now let us consider the general case when a sequence $\left\{b_{n}\right\}_{n \in Z} \in l_{1}$ is concentrated on $Z$. It follows from the condition $\left\{b_{n}\right\}_{n \in Z} \in l_{1}$ that, for any $\varepsilon>0$, there exist a number $m_{\varepsilon} \in N$ satisfying

$$
\sum_{|n|>m_{\varepsilon}}\left|b_{n}\right|<\frac{\varepsilon}{8 C_{0}}
$$

where $C_{0}$ is the constant from (2). Setting

$b_{n}^{\prime}=\left\{\begin{array}{lr}0, & \text { for }|n|>m_{\varepsilon} \\ b_{n}-\frac{1}{2 m_{\varepsilon}+1} \sum_{|k| \leq m_{\varepsilon}} b_{k}, \text { for }|n| \leq m_{\varepsilon}\end{array}, \quad b_{n}^{\prime \prime}= \begin{cases}b_{n}, & \text { for }|n|>m_{\varepsilon} \\ \frac{1}{2 m_{\varepsilon}+1} \sum_{|k| \leq m_{\varepsilon}} b_{k}, & \text { for }|n| \leq m_{\varepsilon}\end{cases}\right.$ 
we have

$$
b_{n}=b_{n}^{\prime}+b_{n}^{\prime \prime}
$$

the sequence $\left\{b_{n}^{\prime}\right\}_{n \in Z} \in l_{1}$ is concentrated on the finite interval $\left[-m_{\varepsilon}, m_{\varepsilon}\right]$,

$$
\begin{aligned}
\sum_{n \in Z} b_{n}^{\prime} & =\sum_{|n| \leq m_{\varepsilon}} b_{n}^{\prime}=\sum_{|n| \leq m_{\varepsilon}}\left[b_{n}-\frac{1}{2 m_{\varepsilon}+1} \sum_{|k| \leq m_{\varepsilon}} b_{k}\right] \\
& =\sum_{|n| \leq m_{\varepsilon}} b_{n}-\frac{1}{2 m_{\varepsilon}+1}\left(2 m_{\varepsilon}+1\right) \sum_{|k| \leq m_{\varepsilon}} b_{k}=0
\end{aligned}
$$

and the sequence $\left\{b_{n}^{\prime \prime}\right\}_{n \in Z} \in l_{1}$ satisfies the inequality

$$
\begin{aligned}
\sum_{n \in Z}\left|b_{n}^{\prime \prime}\right| & =\sum_{|n|>m_{\varepsilon}}\left|b_{n}^{\prime \prime}\right|+\sum_{|n| \leq m_{\varepsilon}}\left|b_{n}^{\prime \prime}\right|=\sum_{|n|>m_{\varepsilon}}\left|b_{n}\right|+\sum_{|n| \leq m_{\varepsilon}}\left|\frac{1}{2 m_{\varepsilon}+1} \sum_{|k| \leq m_{\varepsilon}} b_{k}\right| \\
& =\sum_{|n|>m_{\varepsilon}}\left|b_{n}\right|+\left|\sum_{|k| \leq m_{\varepsilon}} b_{k}\right|=\sum_{|n|>m_{\varepsilon}}\left|b_{n}\right|+\left|\sum_{k \in Z} b_{k}-\sum_{|k|>m_{\varepsilon}} b_{k}\right| \\
& =\sum_{|n|>m_{\varepsilon}}\left|b_{n}\right|+\left|\sum_{|k|>m_{\varepsilon}} b_{k}\right| \leq 2 \sum_{|n|>m_{\varepsilon}}\left|b_{n}\right|<\frac{\varepsilon}{4 C_{0}} .
\end{aligned}
$$

Since the sequence $\left\{b_{n}^{\prime}\right\}_{n \in Z} \in l_{1}$ is concentrated on $\left[-m_{\varepsilon}, m_{\varepsilon}\right]$ and $\sum_{n \in Z} b_{n}^{\prime}=0$, then for the sequence $\left\{b_{n}^{\prime}\right\}_{n \in Z} \in l_{1}$ Eq. (4) is satisfied, and therefore, there exists $\lambda$ $(\varepsilon)>0$ such that, for $0<\lambda<\lambda(\varepsilon)$,

$$
\lambda \tilde{b}^{\prime}\left(\frac{\lambda}{2}\right)<\frac{\varepsilon}{2},
$$

where $\tilde{b}^{\prime}(\lambda)=\sum_{\left\{n \in Z:\left|\tilde{b}_{n}^{\prime}\right|>\lambda\right\}}$ 1. On the other hand, from (2) and (6) it follows that

$$
\lambda \tilde{b^{\prime \prime}}\left(\frac{\lambda}{2}\right) \leq 2 C_{0} \sum_{n \in Z}\left|b_{n}^{\prime \prime}\right|<\frac{\varepsilon}{2}
$$

for any $\lambda>0$, where $\tilde{b^{\prime \prime}}(\lambda)=\sum_{\left\{n \in Z:\left|{\tilde{b^{\prime \prime}}}_{n}\right|>\lambda\right\}} 1$.

Since $\tilde{b}_{n}={\tilde{b^{\prime}}}_{n}+{\tilde{b^{\prime \prime}}}_{n}$ for every $n \in Z$, we have, for every $\lambda>0$,

$$
\left\{n \in Z:\left|\tilde{b}_{n}\right|>\lambda\right\} \subset\left\{n \in Z:\left|\tilde{b}_{n}^{\prime}\right|>\lambda / 2\right\} \cup\left\{n \in Z:\left|\tilde{b}_{n}^{\prime \prime}\right|>\lambda / 2\right\} .
$$


For any $0<\lambda<\lambda(\varepsilon)$ from inequalities (7), (8) and inclusion (9) we get

$$
\tilde{b}(\lambda)=\sum_{\left\{n \in Z:\left|\tilde{b}_{n}\right|>\lambda\right\}} 1 \leq \sum_{\left\{n \in Z:\left|\tilde{b}_{n}^{\prime}\right|>\lambda / 2\right\}} 1+\sum_{\left\{n \in Z:\left|\tilde{b}_{n}^{\prime \prime}\right|>\lambda / 2\right\}} 1=\tilde{b}^{\prime}(\lambda / 2)+\tilde{b}^{\prime \prime}(\lambda / 2)<\frac{\varepsilon}{\lambda} .
$$

This shows that equality (4) is valid for all $\left\{b_{n}\right\}_{n \in Z} \in l_{1}$ satisfying the condition $\sum_{n \in Z} b_{n}=0$. This completes the proof of Lemma 1 .

Proof of Theorem 1 In the case $\sum_{n \in Z} b_{n}=0$ the assertion of the theorem follows from Lemma 1. Let us consider the case $\sum_{n \in Z} b_{n}=\alpha \neq 0$. Puttig

$$
b_{n}^{\prime}=\left\{\begin{array}{ll}
b_{n}, & \text { for } n \neq 0 \\
b_{0}-\alpha, & \text { for } n=0
\end{array}, \quad b_{n}^{\prime \prime}= \begin{cases}0, & \text { for } n \neq 0 \\
\alpha, & \text { for } n=0\end{cases}\right.
$$

we have $b_{n}=b_{n}^{\prime}+b_{n}^{\prime \prime}$ and $\sum_{n \in Z} b_{n}^{\prime}=0$. It follows from Lemma 1 that

$$
\tilde{b^{\prime}}(\lambda)=o\left(\frac{1}{\lambda}\right), \lambda \rightarrow 0+
$$

Since $\tilde{b}_{n}{ }_{n}=\frac{\alpha}{n}$ for $n \neq 0, \tilde{b^{\prime \prime}}{ }_{0}=0$, we have

$$
\tilde{b}^{\prime \prime}(\lambda)=\sum_{\left\{n \in Z:\left|\tilde{b}_{n}^{\prime \prime}\right|>\lambda\right\}} 1=\sum_{\{n \in Z \backslash\{0\}:|\alpha / n|>\lambda\}} 1=\sum_{\{n \in Z \backslash\{0\}:|n|<\alpha / \lambda\}} 1=2[|\alpha / \lambda|],
$$

where $[|\alpha / \lambda|]$ is the integer part of the number $|\alpha / \lambda|$.

Since $\tilde{b}_{n}={\tilde{b^{\prime}}}_{n}+{\tilde{b^{\prime \prime}}}_{n}$ for every $n \in Z$, we have, for any $0<\varepsilon<1$,

$$
\begin{aligned}
& \left\{n \in Z:\left|\tilde{b}_{n}\right|>\lambda\right\} \subset\left\{n \in Z:\left|{\tilde{b^{\prime}}}_{n}\right|>\varepsilon \lambda\right\} \cup\left\{n \in Z:\left|{\tilde{b^{\prime \prime}}}_{n}\right|>(1-\varepsilon) \lambda\right\} \\
& \left\{n \in Z:\left|\tilde{b}_{n}\right|>\lambda\right\} \supset\left\{n \in Z:\left|{\tilde{b^{\prime \prime}}}_{n}\right|>(1+\varepsilon) \lambda\right\} \backslash\left\{n \in Z:\left|\tilde{b}_{n}^{\prime}\right|>\varepsilon \lambda\right\} .
\end{aligned}
$$

Hence

$$
\begin{aligned}
\tilde{b}(\lambda) & =\sum_{\left\{n \in Z:\left|\tilde{b}_{n}\right|>\lambda\right\}} 1 \leq \sum_{\left\{n \in Z:\left|\tilde{b}_{n}^{\prime}\right|>\varepsilon \lambda\right\}} 1+\sum_{\left\{n \in Z:\left|\tilde{b}_{n}^{\prime \prime}\right|>(1-\varepsilon) \lambda\right\}} 1 \\
& =\tilde{b}^{\prime}(\varepsilon \lambda)+\tilde{b}^{\prime \prime}((1-\varepsilon) \lambda), \\
& \tilde{b}(\lambda)=\sum_{\left\{n \in Z:\left|\tilde{b}_{n}\right|>\lambda\right\}} 1 \geq \sum_{\left\{n \in Z:\left|\tilde{b}_{n}^{\prime \prime}\right|>(1+\varepsilon) \lambda\right\}} 1-\sum_{\left\{n \in Z:\left|\tilde{b}_{n}^{\prime}\right|>\varepsilon \lambda\right\}} 1 \\
& =\tilde{b}^{\prime \prime}((1+\varepsilon) \lambda)-\tilde{b}^{\prime}(\varepsilon \lambda) .
\end{aligned}
$$


Now, using (10), (11),

$$
\begin{aligned}
\limsup _{\lambda \rightarrow 0+} \lambda \cdot \tilde{b}(\lambda) & \leq \limsup _{\lambda \rightarrow 0+} \lambda \cdot\left[\tilde{b}^{\prime}(\varepsilon \lambda)+\tilde{b}^{\prime \prime}((1-\varepsilon) \lambda)\right] \\
& =\limsup _{\lambda \rightarrow 0+} \lambda \cdot 2\left[\left|\frac{\alpha}{(1-\varepsilon) \lambda}\right|\right]=\frac{2|\alpha|}{1-\varepsilon},
\end{aligned}
$$

$$
\begin{aligned}
\liminf _{\lambda \rightarrow 0+} \lambda \cdot \tilde{b}(\lambda) & \geq \liminf _{\lambda \rightarrow 0+} \lambda \cdot\left[\tilde{b}^{\prime \prime}((1+\varepsilon) \lambda)-\tilde{b}^{\prime}(\varepsilon \lambda)\right] \\
& =\liminf _{\lambda \rightarrow 0+} \lambda \cdot 2\left[\left|\frac{\alpha}{(1+\varepsilon) \lambda}\right|\right]=\frac{2|\alpha|}{1+\varepsilon} .
\end{aligned}
$$

Since $\varepsilon$ is arbitrary, it follows from (12), (13) that

$$
\liminf _{\lambda \rightarrow 0+} \lambda \cdot \tilde{b}(\lambda)=\limsup _{\lambda \rightarrow 0+} \lambda \cdot \tilde{b}(\lambda)=2|\alpha| .
$$

Hence (3) holds. This completes the proof of Theorem 1.

\section{Q-Summability of Series and the Hilbert Transform}

For a measurable complex function $f$ on an interval $[a, b] \subset R$, we set

$$
\begin{aligned}
& {[f(x)]_{n}=[f(x)]^{n}=f(x) \text { for }|f(x)| \leq n,} \\
& {[f(x)]_{n}=n \cdot \operatorname{sgn} f(x), \quad[f(x)]^{n}=0 \text { for }|f(x)|>n, n \in N,}
\end{aligned}
$$

where $\operatorname{sgn} z=z /|z|$ for $z \neq 0$ and $\operatorname{sgn} 0=0$.

In 1929, Titchmarsh [22] introduced the notions of $Q$-and $Q^{\prime}$-integrals of a function measurable on $[a, b]$.

Definition 1 If the finite limit $\lim _{n \rightarrow \infty} \int_{a}^{b}[f(x)]_{n} d x\left(\lim _{n \rightarrow \infty} \int_{a}^{b}[f(x)]^{n} d x\right.$, respectively) exists, then $f$ is said to be $Q$-integrable ( $Q^{\prime}$-integrable, respectively) on $[a, b]$; that is, $f \in Q[a, b]\left(f \in Q^{\prime}[a, b]\right)$. The value of this limit is referred to as the $Q$-integral ( $Q^{\prime}$-integral) of this function and is denoted by

$$
(Q) \int_{a}^{b} f(x) d x\left(\left(Q^{\prime}\right) \int_{a}^{b} f(x) d x\right)
$$

As in Definition 1, one defines the $Q$ - and $Q^{\prime}$-integrals for a function measurable on the real axis $R$.

Given a measurable complex function $f$ on the real axis $R$, we set

$$
[f(x)]_{\delta, \lambda}=[f(x)]^{\delta, \lambda}=f(x) \text { for } \delta \leq|f(x)| \leq \lambda,
$$




$$
\begin{aligned}
& {[f(x)]_{\delta, \lambda}=[f(x)]^{\delta, \lambda}=0 \text { for }|f(x)|<\delta,} \\
& {[f(x)]_{\delta, \lambda}=\lambda \operatorname{sgn} f(x), \quad[f(x)]^{\delta, \lambda}=0 \text { for }|f(x)|>\lambda, \quad 0<\delta<\lambda .}
\end{aligned}
$$

Definition 2 If the finite limit $\lim _{\delta \rightarrow 0+} \int_{R}[f(x)]_{\delta, \lambda} d x$

$$
\lambda \rightarrow+\infty
$$

(lim $\delta \rightarrow 0+\int_{R}[f(x)]^{\delta, \lambda} d x$, respectively) exists, then $f$ is said to be $Q$-integrable $\lambda \rightarrow+\infty$

( $Q^{\prime}$-integrable, respectively) on $R$; that is, $f \in Q(R)\left(f \in Q^{\prime}(R)\right)$. The value of this limit is referred to as the $Q$-integral ( $Q^{\prime}$-integral) of this function and is denoted by

$$
\text { (Q) } \int_{R} f(x) d x\left(\left(Q^{\prime}\right) \int_{R} f(x) d x\right) \text {. }
$$

A very uncomfortable fact impeding the application of $Q$-integrals and $Q^{\prime}$-integrals when dealing with diverse problems of function theory is the absence of the additivity property; that is, the $Q$-integrability ( $Q^{\prime}$-integrability) of two functions does not imply the $Q$-integrability ( $Q^{\prime}$-integrability) of their sum. If one adds the conditions

$$
\begin{gathered}
\delta m\{x \in R:|f(x)|>\delta\}=o(1), \quad \delta \rightarrow 0+, \\
\lambda m\{x \in R:|f(x)|>\lambda\}=o(1), \quad \lambda \rightarrow+\infty,
\end{gathered}
$$

to the definition of $Q$-integrability ( $Q^{\prime}$-integrability) of a function $f$, then the $Q$-integral and $Q^{\prime}$-integral coincide $\left(Q(R)=Q^{\prime}(R)\right)$ and these integrals become additive.

Definition 3 If $f \in Q^{\prime}(R)$ (or $f \in Q(R)$ ) and conditions (14) and (15) holds, then $f$ is said to be $A$-integrable on $R$; that is, $f \in A(R)$. In this case, the limit $\lim _{\delta \rightarrow 0+} \int_{R}[f(x)]^{\delta, \lambda} d x$ (or the limit $\lim _{\delta \rightarrow 0+} \int_{R}[f(x)]_{\delta, \lambda} d x$ ) is denoted by

$$
\lambda \rightarrow+\infty \quad \lambda \rightarrow+\infty
$$

$$
\text { (A) } \int_{R} f(x) d x \text {. }
$$

Properties of $Q$ - and $Q^{\prime}$-integrals were investigated in [2, 3, 6, 11, 12, 22]; for the applications of $A$-, $Q$ - and $Q^{\prime}$-integrals in the theory of functions of real and complex variables we refer the reader to [1-6, 19, 20, 22, 23].

We need the following theorem proved in [3] and [4].

Theorem A [4, Theorem 4] Let $v$ be a finite complex measure on the real axis $R$. Then

$$
\left(Q^{\prime}\right) \int_{R}(H v)(x) d x=0,
$$


where $(H v)(x)=\frac{1}{\pi} \int_{R} \frac{d v(t)}{x-t}$ is the Hilbert transform of the measure $v$.

Definition 4 We denote by $M(R ; C)$ the class of measurable complex-valued functions $f$ on $R$ for which the finite limits $\lim _{\lambda \rightarrow+\infty} \lambda m\{z \in R:|f(z)|>\lambda\}$ and $\lim _{\delta \rightarrow 0+} \delta m\{z \in R:|f(z)|>\delta\}$ exist.

Remark 1 Note that the Hilbert transform of a finite complex measure belong to the class of functions $M(R ; C)$ (see [4]).

Theorem B [3, Theorem 2.3] If a function $f \in M(R ; C)$ is $Q^{\prime}$-integrable on $R$ and a function $g$ is $A$-integrable on $R$, then their sum $f+g \in M(R ; C)$ is $Q^{\prime}$-integrable on $R$, and

$$
\left(Q^{\prime}\right) \int_{R}[f(x)+g(x)] d x=\left(Q^{\prime}\right) \int_{R} f(x) d x+(A) \int_{R} g(x) d x .
$$

Similar to the definition of the $Q$-integral, we define the $Q$-sum of series. Let $\left\{a_{n}\right\}_{n \in Z}$ be a sequence of complex numbers.

Definition 5 If the finite $\operatorname{limit}_{\lim _{\lambda \rightarrow 0+}} \sum_{\left\{n \in Z:\left|a_{n}\right| \geq \lambda\right\}} a_{n}$ exists, then the series $\sum_{n \in Z} a_{n}$ is said to be $Q$-summable, and the value of this limit is referred to as the $Q$-sum of this series and is denoted by

$$
\text { (Q) } \sum_{n \in Z} a_{n}
$$

$Q$-summable series does not enjoy the additivity property; that is, the $Q$-summability of two series does not imply the $Q$-summability of their sum. If one adds the condition

$$
\sum_{\left\{n \in Z:\left|a_{n}\right|>\lambda\right\}} 1=o\left(\frac{1}{\lambda}\right), \lambda \rightarrow 0+
$$

to the definition of $Q$-summability of a series $\sum_{n \in Z} a_{n}$, then the $Q$-sum become additive.

Definition 6 If a series $\sum_{n \in Z} a_{n}$ is $Q$-summable and condition (16) holds, then the series $\sum_{n \in Z} a_{n}$ is said to be $A$-summable, and the $\operatorname{limit}_{\lambda \rightarrow 0+} \sum_{\left\{n \in Z:\left|a_{n}\right| \geq \lambda\right\}} a_{n}$ is denoted in this case by $(A) \sum_{n \in Z} a_{n}$.

Theorem 2 Let $\left\{b_{n}\right\}_{n \in Z} \in l_{1}$. Then the series $\sum_{n \in Z} \tilde{b}_{n}$ is $Q$-summable and the equation

$$
\text { (Q) } \sum_{n \in Z} \tilde{b}_{n}=0
$$

holds.

Proof of Theorem 2 Define the function $f(x)$ to be $2 \pi b_{n}$ for $x \in[n-1 / 4, n+1 / 4]$, $n \in Z$ and 0 elsewhere, the function $F(x)$ to be $\tilde{b}_{n}$ for $x \in[n-1 / 2, n+1 / 2), n \in Z$ and 


$$
G(x)=(H f)(x)-F(x) .
$$

We first show that $G_{1}(x) \in L_{1}(R)$.

For every $x \in[n-1 / 2, n+1 / 2), x \neq n \pm 1 / 4$ we have

$$
\begin{aligned}
G(x) & =\frac{1}{\pi} \int_{R} \frac{f(t)}{x-t} d t-\tilde{b}_{n}=\frac{1}{\pi} \sum_{m \in Z_{m}} \int_{m-1 / 4}^{m+1 / 4} \frac{2 \pi b_{m}}{x-t} d t-\sum_{m \neq n} \frac{b_{m}}{n-m} \\
& =\left(\sum_{m \neq n} 2 b_{m} \int_{m-1 / 4}^{m+1 / 4} \frac{d t}{x-t}+2 b_{n} \int_{n-1 / 4}^{n+1 / 4} \frac{d t}{x-t}\right)-\sum_{m \neq n} \frac{b_{m}}{n-m} \\
& =\sum_{m \neq n} 2 b_{m}\left(\int_{m-1 / 4}^{m+1 / 4}\left(\frac{1}{x-t}-\frac{1}{n-m}\right) d t\right)+2 b_{n} \int_{n-1 / 4}^{n+1 / 4} \frac{d t}{x-t}=G_{1}(x)+G_{2}(x) .
\end{aligned}
$$

Let $m \neq n$. Then, for every $x \in[n-1 / 2, n+1 / 2)$ and $t \in[m-1 / 4, m+1 / 4]$, since

$$
|x-n| \leq 1 / 2, \quad|m-t| \leq 1 / 4, \quad|x-t| \geq|n-m|-|x-n|-|m-t| \geq|n-m|-3 / 4
$$

we get

$$
\left|\frac{1}{x-t}-\frac{1}{n-m}\right|=\frac{|n-x+t-m|}{|x-t| \cdot|n-m|} \leq \frac{1 / 2+1 / 4}{|n-m| \cdot(|n-m|-3 / 4)}=\frac{3}{|n-m| \cdot(4|n-m|-3)} .
$$

Therefore, for every $x \in[n-1 / 2, n+1 / 2)$,

$$
\left|G_{1}(x)\right| \leq \sum_{m \neq n} 2\left|b_{m}\right| \cdot \int_{m-1 / 4}^{m+1 / 4}\left|\frac{1}{x-t}-\frac{1}{n-m}\right| d t \leq \sum_{m \neq n} 3\left|b_{m}\right| \cdot \frac{1}{|n-m| \cdot(4|n-m|-3)} .
$$

From inequality (20) it follows that

$$
\int_{R}\left|G_{1}(x)\right| d x=\sum_{n \in Z_{n}} \int_{-1 / 2}^{n+1 / 2}\left|G_{1}(x)\right| d x \leq \sum_{n \in Z} \sum_{m \neq n} 3\left|b_{m}\right| \cdot \frac{1}{|n-m| \cdot(4|n-m|-3)} .
$$

Since for every $m \in Z$ the series $\sum_{n \neq m} \frac{1}{|n-m| \cdot(4|n-m|-3)}=\sum_{k \neq 0} \frac{1}{|k| \cdot(4|k|-3)}$ is convergent, we have from (21) 


$$
\begin{aligned}
& \int_{R}\left|G_{1}(x)\right| d x \leq \sum_{m \in Z} 3\left|b_{m}\right| \sum_{n \neq m} \frac{1}{|n-m| \cdot(4|n-m|-3)} \\
& \quad=3 \sum_{m \in Z}\left|b_{m}\right| \cdot \sum_{k \neq 0} \frac{1}{|k| \cdot(4|k|-3)}=3 \sum_{k \neq 0} \frac{1}{|k| \cdot(4|k|-3)} \cdot \sum_{m \in Z}\left|b_{m}\right|
\end{aligned}
$$

and, therefore, $G_{1}(x) \in L_{1}(R)$.

Let us show that $G_{2}(x) \in L_{1}(R)$.

For every $n \in Z$ we subdivide the set $[n-1 / 2, n+1 / 2) \backslash\{n-1 / 4 ; n+1 / 4\}$ into four parts: $[n-1 / 2, n-1 / 4),(n-1 / 4, n],(n, n+1 / 4),(n+1 / 4, n+1 / 2)$.

If $x \in[n-1 / 2, n-1 / 4)$, then

$$
G_{2}(x)=2 b_{n} \int_{n-1 / 4}^{n+1 / 4} \frac{d t}{x-t}=2 b_{n}[-\ln (n+1 / 4-x)+\ln (n-1 / 4-x)]
$$

For every $x \in R$ and $\delta>0$, the equality

$$
\text { v.p. } \int_{x-\delta}^{x+\delta} \frac{d t}{x-t}=\text { v.p. } \int_{-\delta}^{\delta} \frac{d u}{-u}=0
$$

holds. Therefore, if $x \in(n-1 / 4, n]$, then

$$
\begin{aligned}
& G_{2}(x)=2 b_{n} \text { v.p. } \int_{n-1 / 4}^{n+1 / 4} \frac{d t}{x-t}=2 b_{n}\left(\text { v.p. } \int_{x-(x-n+1 / 4)}^{x+(x-n+1 / 4)} \frac{d t}{x-t}+\int_{x+(x-n+1 / 4)}^{n+1 / 4} \frac{d t}{x-t}\right) \\
& =2 b_{n} \int_{2 x-n+1 / 4}^{n+1 / 4} \frac{d t}{x-t}=2 b_{n}[-\ln (n+1 / 4-x)+\ln (x-n+1 / 4)]
\end{aligned}
$$

if $x \in(n, n+1 / 4)$, then

$$
\begin{gathered}
G_{2}(x)=2 b_{n} \text { v.p. } \int_{n-1 / 4}^{n+1 / 4} \frac{d t}{x-t}=2 b_{n}\left(\text { v.p. } \int_{n-1 / 4}^{x-(n+1 / 4-x)} \frac{d t}{x-t}+\int_{x-(n+1 / 4-x)}^{x+(n+1 / 4-x)} \frac{d t}{x-t}\right) \\
=2 b_{n} \int_{n-1 / 4}^{2 x-n-1 / 4} \frac{d t}{x-t}=2 b_{n}[-\ln (n+1 / 4-x)+\ln (x-n+1 / 4)] ;
\end{gathered}
$$


if $x \in(n+1 / 4, n+1 / 2)$, then

$$
G_{2}(x)=2 b_{n} \int_{n-1 / 4}^{n+1 / 4} \frac{d t}{x-t}=2 b_{n}[-\ln (x-n-1 / 4)+\ln (x-n+1 / 4)] .
$$

This shows that for every $x \in[n-1 / 2, n+1 / 2), x \neq n \pm 1 / 4$ we have

$$
G_{2}(x)=2 b_{n}[-\ln |x-n-1 / 4|+\ln |x-n+1 / 4|]
$$

and, therefore,

$$
\left|G_{2}(x)\right| \leq\left|2 b_{n}\right|\left[\ln \frac{1}{|x-n-1 / 4|}+\ln \frac{1}{|x-n+1 / 4|}\right] .
$$

Now, for every $n \in Z$,

$$
\int_{n-1 / 2}^{n+1 / 2}\left|G_{2}(x)\right| d x \leq\left|2 b_{n}\right| \cdot M_{1}
$$

where $M_{1}=\int_{n-1 / 2}^{n+1 / 2}\left[\ln \frac{1}{|x-n-1 / 4|}+\ln \frac{1}{|x-n+1 / 4|}\right] d x=\int_{-1 / 2}^{1 / 2}\left[\ln \frac{1}{|u-1 / 4|}+\right.$ $\left.\ln \frac{1}{|u+1 / 4|}\right] d u$. Therefore,

$$
\int_{R}\left|G_{2}(x)\right| d x=\sum_{n \in Z} \int_{n-1 / 2}^{n+1 / 2}\left|G_{2}(x)\right| d x \leq 2 M_{1} \sum_{n \in Z}\left|b_{n}\right| .
$$

It follows that $G_{2}(x) \in L_{1}(R)$, and hence $G(x) \in L_{1}(R)$.

Now we prove that the series $\sum_{n \in Z} \tilde{b}_{n}$ is $Q$-summable and Eq. (17) holds.

Since $F(x)=(H f)(x)-G(x), H f \in M(R ; C)$ (see: Remark 1) and $G(x) \in L_{1}$ $(R)$, it follows from Theorems A and B that the function $F(x)$ is $Q^{\prime}$-integrable on $R$, and moreover,

$$
\left(Q^{\prime}\right) \int_{R} F(x) d x=\left(Q^{\prime}\right) \int_{R}(H f)(x) d x-\int_{R} G(x) d x=-\int_{R} G(x) d x
$$

The function $F(x)$ is bounded and by definition for every $\lambda>0$

$$
\{x \in R:|F(x)|>\lambda\}=\underset{n \in Z}{\cup}\left\{x \in[n-1 / 2, n+1 / 2):\left|\tilde{b}_{n}\right|>\lambda\right\}=\underset{\left\{n:\left|\tilde{b}_{n}\right|>\lambda\right\}}{\cup}[n-1 / 2, n+1 / 2),
$$


hence

$$
\begin{gathered}
\left(Q^{\prime}\right) \int_{R} F(x) d x=\lim _{\substack{\lambda \rightarrow 0+\\
\delta \rightarrow+\infty}} \int_{\{x \in R: \lambda<|F(x)|<\delta\}} F(x) d x=\lim _{\lambda \rightarrow 0+} \int_{\{x \in R:|F(x)|>\lambda\}} F(x) d x \\
=\lim _{\lambda \rightarrow 0+} \sum_{\left\{n:\left|\tilde{b}_{n}\right|>\lambda\right\}} \int_{n-1 / 2}^{n+1 / 2} \tilde{b}_{n} d x=\lim _{\lambda \rightarrow 0+} \sum_{\left\{n:\left|\tilde{b}_{n}\right|>\lambda\right\}} \tilde{b}_{n}=(Q) \sum_{n \in Z} \tilde{b}_{n} .
\end{gathered}
$$

It follows from (23) and (24) that the series $\sum_{n \in Z} \tilde{b}_{n}$ is $Q$-summable and the equation

$$
\text { (Q) } \sum_{n \in Z} \tilde{b}_{n}=-\int_{R} G(x) d x \text {. }
$$

holds. For every $n \in Z$ it follows from (22) that

$$
\begin{aligned}
& \int_{n-1 / 2}^{n+1 / 2} G_{2}(x) d x=2 b_{n}\left[-\int_{n-1 / 2}^{n+1 / 2} \ln |x-n-1 / 4| d x+\int_{n-1 / 2}^{n+1 / 2} \ln |x-n+1 / 4| d x\right] \\
& =2 b_{n}\left[-\int_{-1 / 2}^{1 / 2} \ln |u-1 / 4| d u+\int_{-1 / 2}^{1 / 2} \ln |u+1 / 4| d u\right] \\
& =2 b_{n}\left[\int_{-1 / 2}^{1 / 2} \ln |u-1 / 4| d(-u)+\int_{-1 / 2}^{1 / 2} \ln |u+1 / 4| d u\right] \\
& =2 b_{n}\left[-\int_{-1 / 2}^{1 / 2} \ln |-z-1 / 4| d z+\int_{-1 / 2}^{1 / 2} \ln |u+1 / 4| d u\right]=0 .
\end{aligned}
$$

Therefore,

$$
\int_{R} G_{2}(x) d x=\sum_{n \in Z_{n-1 / 2}} \int_{n+1 / 2} G_{2}(x) d x=0 .
$$

By (19) for every $m \in Z$ and $t \in[m-1 / 4, m+1 / 4]$ the series $\sum_{n \neq m} \int_{n-1 / 2}^{n+1 / 2}\left|\frac{1}{x-t}-\frac{1}{n-m}\right| d x$ is convergent. Hence 


$$
\begin{aligned}
& \sum_{n \neq m} \int_{n-1 / 2}^{n+1 / 2}\left(\frac{1}{x-t}-\frac{1}{n-m}\right) d x \\
& \quad=\lim _{p \rightarrow \infty}\left(\sum_{n=m-p_{n-1 / 2}}^{m-1} \int_{n+1 / 2}^{n+1 / 2}\left(\frac{1}{x-t}-\frac{1}{n-m}\right) d x+\sum_{n=m+1}^{m+p} \int_{n-1 / 2}^{n+1 / 2}\left(\frac{1}{x-t}-\frac{1}{n-m}\right) d x\right)
\end{aligned}
$$

Since for every $m \in Z$ and $p \in N$

$$
\begin{aligned}
\sum_{n=m-p}^{m-1} \frac{1}{n-m}+\sum_{n=m+1}^{m+p} \frac{1}{n-m}= & \left(-\frac{1}{p}-\frac{1}{p-1}-\cdots-\frac{1}{2}-1\right) \\
& +\left(1+\frac{1}{2}+\cdots+\frac{1}{p-1}+\frac{1}{p}\right)=0
\end{aligned}
$$

it follows from (27) that

$$
\begin{aligned}
& \sum_{n \neq m} \int_{n-1 / 2}^{n+1 / 2}\left(\frac{1}{x-t}-\frac{1}{n-m}\right) d x \\
& =\lim _{p \rightarrow \infty}\left(\sum_{n=m-p_{n-1 / 2}}^{m-1} \int_{n+t}^{n+1 / 2} \frac{1}{x-t} d x+\sum_{n=m+1}^{m+p} \int_{n-1 / 2}^{n+1 / 2} \frac{1}{x-t} d x\right)=\lim _{p \rightarrow \infty}\left(\int_{m-p-1 / 2}^{m-1 / 2} \frac{1}{x-t} d x+\int_{m+1 / 2}^{m+p+1 / 2} \frac{1}{x-t} d x\right) \\
& =\lim _{p \rightarrow \infty}[\ln (t-m+1 / 2)-\ln (t-m+p+1 / 2)+\ln (m+p+1 / 2-t)-\ln (m+1 / 2-t)] \\
& =\ln (t-m+1 / 2)-\ln (m+1 / 2-t) .
\end{aligned}
$$

Therefore, for every $m \in Z$,

$$
\begin{aligned}
& \int_{m-1 / 4}^{m+1 / 4}\left[\sum_{n \neq m} \int_{n-1 / 2}^{n+1 / 2}\left(\frac{1}{x-t}-\frac{1}{n-m}\right) d x\right] d t=\int_{m-1 / 4}^{m+1 / 4}[\ln (t-m+1 / 2)-\ln (m+1 / 2-t)] d t \\
& =\int_{-1 / 4}^{1 / 4}[\ln (u+1 / 2)-\ln (1 / 2-u)] d u=\int_{-1 / 4}^{1 / 4} \ln (u+1 / 2) d u+\int_{-1 / 4}^{1 / 4} \ln (1 / 2-u) d(-u) \\
& =\int_{-1 / 4}^{1 / 4} \ln (u+1 / 2) d u-\int_{-1 / 4}^{1 / 4} \ln (1 / 2+z) d z=0 .
\end{aligned}
$$

Now it follows from Fubini's theorem that

$$
\begin{aligned}
& \int_{R} G_{1}(x) d x=\sum_{n \in Z} \int_{n-1 / 2}^{n+1 / 2} G_{1}(x) d x=\sum_{n \in Z} \int_{n-1 / 2}^{n+1 / 2}\left[\sum_{m \neq n} 2 b_{m} \int_{m-1 / 4}^{m+1 / 4}\left(\frac{1}{x-t}-\frac{1}{n-m}\right) d t\right] d x \\
& =\sum_{m \in Z} 2 b_{m}\left[\int_{m-1 / 4}^{m+1 / 4}\left[\sum_{n \neq m} \int_{n-1 / 2}^{n+1 / 2}\left(\frac{1}{x-t}-\frac{1}{n-m}\right) d x\right] d t\right]=0 .
\end{aligned}
$$


Now from Eqs. (18), (25), (26) and (28) we finally obtain (17). This completes the proof of theorem 2 .

Acknowledgements The authors are very grateful to the referee for many useful suggestions and comments that improved the original manuscript. The research of the first author was supported by the Science Development Foundation under the President of the Republic of Azerbaijan (Grant No. EIF/MQM/ElmTehsil-1-2016-1(26)-71/08/01).

\section{References}

1. Aleksandrov, A.B.: A-integrability of the boundary values of harmonic functions. Math. Notes 30(1), 515-523 (1981)

2. Aliev, R.A.: $N^{ \pm}$-integrals and boundary values of Cauchy-type integrals of finite measures. Sbornik Math. 205(7), 913-935 (2014)

3. Aliev, R.A.: On the properties of $Q$ - and $Q^{\prime}$-integrals of the function measurable on the real axis. Proc. Inst. Math. Mech. NAS Azerb. 41(1), 56-62 (2015)

4. Aliev, R.A.: On properties of Hilbert transform of finite complex measures. Complex Anal. Oper. Theory 10(1), 171-185 (2016)

5. Aliev, R.A.: On Laurent coefficients of Cauchy type integrals of finite complex measures. Proc. Inst. Math. Mech. NAS Azerb. 42(2), 292-303 (2016)

6. Aliev, R.A.: Representability of Cauchy-type integrals of finite complex measures on the real axis in terms of their boundary values. Complex Var. Elliptic Equ. 62(4), 536-553 (2017)

7. Andersen, K.F.: Inequalities with weights for discrete Hilbert transforms. Can. Math. Bull. 20, 9-16 (1977)

8. Belov, Y., Mengestie, T.Y., Seip, K.: Discrete Hilbert transforms on sparse sequences. Proc. Lond. Math. Soc. 103(1), 73-105 (2011)

9. Belov, Y., Mengestie, T.Y., Seip, K.: Unitary discrete hilbert transforms. J. Anal. Math. 112, 383-393 (2010)

10. De Carli, L., Samad, S.: One-parameter groups and discrete Hilbert transform. Can. Math. Bull. 59, 497-507 (2016)

11. Efimova, M.P.: On the properties of the $Q$-integral. Math. Notes 90(3), 322-332 (2011)

12. Efimova, M.P.: The sufficient condition for integrability of a generalized $Q$-integral and points of integrability. Mosc. Univ. Math. Bull. 70(4), 181-184 (2015)

13. Gabisoniya, I., Meskhi, A.: Two weighted inequalities for a discrete Hilbert transform. Proc. A Razmadze Math. Inst. 116, 107-122 (1998)

14. Hunt, R., Muckenhoupt, B., Wheeden, R.: Weighted norm inequalities for the conjugate function and Hilbert transform. Trans. Am. Math. Soc. 176(2), 227-251 (1973)

15. Laeng, E.: Remarks on the Hilbert transform and some families of multiplier operators related to it. Collect. Math. 58(1), 25-44 (2007)

16. Liflyand, E.: Weighted estimates for the discrete hilbert transform. In: Ruzhansky, M., Tikhonov, S. (eds.) Methods of Fourier Analysis and Approximation Theory. Applied and Numerical Harmonic Analysis, pp. 59-69. Birkhäuser, Cham (2016)

17. Rakotondratsimba, Y.: Two weight inequality for the discrete Hilbert transform. Soochow J. Math. 25(4), 353-373 (1999)

18. Riesz, M.: Sur les fonctions conjuguees. Math. Z. 27, 218-244 (1928)

19. Salimov, T.S.: The A-integral and boundary values of analytic functions. Math. USSR Sb. 64(1), 23-39 (1989)

20. Skvortsov, V.A.: A-integrable martingale sequences and Walsh series. Izvestia Math. 65(3), 607-617 (2001)

21. Stepanov, V.D., Tikhonov, SY.: Two weight inequalities for the Hilbert transform of monotone functions. Dokl. Math. 83(2), 241-242 (2011)

22. Titchmarsh, E.C.: On conjugate functions. Proc. Lond. Math. Soc. 9, 49-80 (1929)

23. Ul'yanov, P.L.: "Integrals of Cauchy type" collection of papers dedicated to the 60th birthday of academician Mikhail Alekseevich Lavrent'ev. Am. Math. Soc. Transl. Ser. 44(2), 129-150 (1965) 
Publisher's Note Springer Nature remains neutral with regard to jurisdictional claims in published maps and institutional affiliations. 